\title{
EVOLUCIÓN DE PARÁMETROS HEMÁTICOS DE TERNEROS MEDIA SANGRE CEBÚ EN CRECIMIENTO
}

\author{
Coppo, José A. y Mussart, Norma B. \\ Cátedra de Fisiología, Facultad de Ciencias Veterinarias, UNNE, \\ Sargento Cabral 2139, Corrientes (3400), Argentina. E-mail: jcoppo@vet.unne.edu.ar
}

\begin{abstract}
RESUMEN
El propósito del trabajo fue obtener intervalos de confianza y detectar variaciones ontogénicas de algunos parámetros hemáticos de interés diagnóstico que pudieran ocurrir entre los 2 y 6 meses de vida del ternero media sangre cebú mantenido en lactación sobre pasturas naturales del nordeste argentino. En cada uno de cuatro años sucesivos, fueron estudiados 15 terneros clínicamente sanos de 60-75 días de edad y 60-90 $\mathrm{kg}$ de peso $(n=60)$. Las determinaciones hemáticas y bioquimicas fueron realizadas con técnicas usuales de laboratorio. Bajo un diseño completamente aleatorizado, se efectuaron estadísticas descriptivas paramétricas y análisis de la variancia a una vía, estableciéndose un riesgo alfa del $5 \%$. Al final del ensayo se verificaron aumentos significativos de cortisol, creatinfosfoquinasa, volumen corpuscular medio, hemoglobina corpuscular media, concentración de hemoglobina corpuscular media, neutrófilos, eosinófilos, urea, proteínas totales, beta y gamma globulinas, así como disminuciones significativas de aldosterona, fructosamina, glucosa, triglicéridos, colesterol total, fósforo inorgánico, fosfatasa alcalina, relación albúmina / globulinas, leucocitos totales y linfocitos.
\end{abstract}

Palabras claves: ternero cruza cebú, crecimiento, valores hemáticos.

\section{SUMMARY}

The purpose of the trial was to obtain confidence intervals and to detect ontogenetic changes of some blood parameters for diagnoses use, happened between 2 and 6 months of life from half-bred Zebu nursling calves maintained on natural pastures in Argentine northeastern. In each one of four successive years, fifteen clinically healthy calves from 60 75 days old and $60-90 \mathrm{~kg}$ liveweight $(n=60)$, were studied. Hematological and biochemical determinations were carried out by conventional laboratory techniques. Using a complete randomized design, parametric descriptive statistics and one-way analysis of the variance, were made. An alpha risk from $5 \%$ was fixed. Significant increases of cortisol, creatine phosphokinase, mean corpuscular volume, mean corpuscular hemoglobin, mean corpuscular hemoglobin concentration, neutrophils, eosinophils, urea, total protein, beta and gamma globulins, as well as significant decreases of aldosterone, fructosamine, glucose, triglycerides, total cholesterol, inorganic phosphorus, alkaline phosphatase, albumin/globulin ratio, total leukocytes and lymphocytes, were verified at the end of the rehearsal.

Key words: half-bred Zebu calf, growth, blood values.

\section{INTRODUCCIÓN}

Los cambios ontogénicos de los parámetros hemáticos de terneros cruza cebú no están suficientemente estudiados en el nordeste argentino. Siendo la Ontogenia el capítulo de la Fisiologia que estudia los cambios estructurales que ocurren durante las distintas etapas de la vida de un mismo individuo, es dable destacar que tales variaciones son desencadenadas tanto por la dinámica interna (filogenética) como por el medio externo (hábitat). Ello genera la necesidad de disponer de valores de referencia regionales que reflejen adecuadamente, para cada estadio ontogénico, la interacción del animal con el entorno geográfico, clima, alimentación y sistema de manejo a que está sometido (Foote et al., 2007).

Un valor de referencia es el obtenido por medición de un tipo particular de magnitud en cierto número de individuos de una población de referencia. Solo tendrá validez cuando para su obtención se hayan estipulado correctamente los criterios de inclusión y exclusión (edad, sexo, raza, lugar geográfico, alimentación). También requiere una detallada descripción de las condiciones fisiológicas y ambientales de la población bajo estudio, la forma de extraer la muestra, el método analítico utilizado y la técnica estadistica aplicada (Solberg, 1992) . 
La estimación estadística de los valores hemáticos "normales" de una población puede realizarse calculando los intervalos de confianza (IC) de una muestra, obteniéndose un rango de dos valores entre los cuales estará contenido el parámetro, con un nivel de confianza fijado de antemano (p.ej. 95\%), que es inverso al riesgo de error previsto $(5 \%)$. Tal nivel de confianza es el utilizado habitualmente en bioquínica clínica, ya que los intervalos más largos (p.ej. 99\%) serán más confiables pero menos precisos y viceversa (Steel y Torrie, 1992).

Para tener valor clínico, cualquier parámetro debe ser comparado con el "valor normal" para la edad, sexo, raza, lugar geográfico y otras variables (Ash, 1980). Surge entonces que los intervalos de referencia son indispensables para efectuar un correcto diagnóstico. Los principales parámetros hemáticos de utilidad diagnóstica en terneros son los obtenidos a partir de eritrogramas, leucogramas, ionogramas, proteinogramas, enzimogramas, glucogramas, lipidogramas y determinaciones hormonales (Dürr, 1980). Los intervalos de referencia disponibles han sido obtenidos a partir de terneros de razas europeas, principalmente en el hemisferio norte (Dürr, 1980; Kolb, 1987; Kaneko, 1989; Jain, 1993).

El objetivo del trabajo fue establecer el intervalo de confianza y las variaciones ontogénicas registradas entre los 2 y 6 meses de edad (temporada estival), para los principales parámetros hemáticos de interés diagnóstico en terneros media sangre cebú clínicamente sanos, mantenidos en lactación al pie de madre sobre pasturas naturales del Nordeste Argentino.

\section{MATERIALES Y MÉTODOS}

Diseño experimental. Sesenta unidades experimentales fueron estudiadas bajo un diseño prospectivo completamente aleatorizado. En cada uno de 4 años consecutivos se emplearon 15 terneros lactantes al pie de la madre, media sangre Nelore $x$ Hereford (60-75 días de edad y $60-90 \mathrm{~kg}$ de peso vivo), $50 \%$ hembras y $50 \%$ machos castrados, clinicamente sanos y fenotípicamente homogéneos, desparasitados y vacunados acorde al manejo sanitario habitual del establecimiento, ubicado en el noroeste de la Provincia de Corrientes. Durante los ensayos los terneros fueron mantenidos sobre pastura natural, que en dicha zona habitualmente posee $6 \%$ de proteína bruta, $2-3 \%$ de grasa, 30 $40 \%$ de extracto no azoado, $25-35 \%$ de fibra bruta y $10 \%$ de cenizas (Peruchena, 1992). La edad fue considerada como variable independiente, en tanto que el peso y los valores hemáticos operaron como variables dependientes cuantitativas continuas, siendo determinados en los días 0 (60-75 días de edad) y 120 (180-195 dias de edad), desde finales de primavera hasta finales de verano (destete).
Toma de muestras. La sangre fue extraida en ayunas, por venopunción yugular, en horario uniforme (7-8 h AM), momento en que los animales fueron pesados en una báscula. Una alícuota de sangre fue tratada con anticoagulante (EDTA) y la otra fue centrifugada $(700 \mathrm{~g}, 10 \mathrm{~min})$ para obtener suero, el cual fue conservado a $4^{\circ} \mathrm{C}$ hasta su procesamiento en laboratorio, efectuado dentro de las 6 horas post-extracción a efectos de evitar alteraciones atribuibles al lapso de almacenamiento.

Pruebas de laboratorio. Con técnicas convencionales (Coppo, 2001), usando reactivos Wiener, Randox, Biopur, Boehringer y DPC-Lab, se determinaron las concentraciones séricas de cortisol (enzimoinmunoensayo por quimioluminiscencia), aldosterona (radioinmunoanálisis por técnica competitiva), fructosamina (nitrotetrazolio), glucosa (glucosa-oxidasa), triglicéridos (lipasa-peroxidasa), colesterol total (colesterol-oxidasa), colesterol ligado a lipoproteínas de alta densidad (C-HDL) y baja densidad (C-LDL) por precipitación selectiva de la lipoproteina y valoración enzimática del colesterol, lipoproteínas alfa y beta (electroforesis en gel de agarosa y densitometria), proteinas totales (biuret), albúminas y globulinas alfa, beta y gamma (electroforesis en acetato de celulosa y densitometria), relación albúminas/globulinas (cálculo), urea (ureasa), fosfatasa alcalina ALP ( $p$-nitrofeniIfosfato), gamma glutamiltransferasa GGT (p-nitroanilida), creatinfosfokinasa CPK (ATP-cisteina), lactato dehidrogenasa LDH (NADH-piruvato), aspartato aminotransferasa AST (NADH-oxoglutarato), cobre (batocuproína), magnesio (calmagita), calcio (cresolftaleincomplexona), fósforo inorgánico (molibdato-ascorbato), hierro (PBTS) y cloro (tiocianato). Sodio y potasio fueron valorados por fotometría de Ilama. El control de calidad de las determinaciones bioquímicas se realizó con patrones liofilizados ad-hoc. Leucocitos, eritrocitos, hemoglobina, hematocrito, volumen corpuscular medio (VCM), hemoglobina corpuscular media (HCM) y concentración de hemoglobina corpuscular media (CHCM) fueron determinados en un analizador hematológico electrónico calibrado con estándares bovinos (Coppo, 1999). La fórmula leucocitaria absoluta se obtuvo por microscopia, a través del recuento diferencial de glóbulos blancos en frotis coloreados con Giemsa.

Cálculos estadísticos. La distribución normal fue constatada mediante el test de Wilk-Shapiro (WS). Las estadísticas descriptivas comprendieron medidas de tendencia central (media aritmética, $\bar{x}$ ), dispersión (desvío estándar, DE) y riesgo (intervalos de confianza, $\mathrm{IC} \pm 95 \%$ ). El análisis de la variancia (ANOVA) a una via se realizó con el auxilio de un software (Statistix), previa verificación de la 
homogeneidad de la variancia por test de Bartlett (Steel y Torrie, 1992). Algunas variables fueron marginadas por el diseño, como los cambios postprandiales (suprimidos por el ayuno previo), el ritmo circadiano (soslayado por el horario matutino uniforme de extracción de muestras), raza y edad (similares). Otros factores de variación (año de ensayo, peso inicial, sexo) fueron tratados como covariables (Solberg, 1992). Para todas las inferencias se estipuló un riesgo alfa del $5 \%$, por debajo del cual se rechazó la hipótesis nula de igualdad.

\section{RESULTADOS Y DISCUSIÓN}

Todos los valores registraron distribución normal (WS), habilitando el uso de estadísticas paramétricas (Steel y Torrie, 1992)). Las diferencias atribuibles al sexo de los terneros, peso inicial y año de ensayo resultaron no significativas.

En Tabla 1 se expone la evolución del peso y valores del hemograma (eritro y leucograma) obtenidos para la totalidad de animales estudiados. La ganancia de peso obtenida por los terneros (666 g/animal/dia) no se aleja de las medias reportadas en la zona para terneros en lactación mantenidos sobre pastura natural, de 707 (Peruchena, 1996) y $663 \mathrm{~g} / \mathrm{animal} / \mathrm{dia}$ (Zehentner, 1996).

La ontogenia provocó leve disminución de los eri- trocitos y aumentos significativos del VCM, cambios que se tradujeron en elevación del hematocrito. Por su parte, aunque el aumento de hemoglobina fue escaso, se elevaron las tasas de CHCM (ligeramente) y HCM (considerablemente), debido al mencionado aumento del VCM. Tales cambios coinciden con la mayoría de los datos bibliográficos, con excepción del hematocrito, dado que algunos autores reportan que en razas lecheras desciende con el crecimiento (Jain, 1993). En terneros para carne de razas europeas (2-3 meses de edad) se hallaron concentraciones de eritrocitos de $9.5 \pm 1.0 \mathrm{~T} / 1$ (Jain, 1993) y 5.0 a $8.7 \mathrm{~T} / 1$ (Dürr, 1980); hematocritos de $22-44 \%$ (Dürr, 1980) y 36\% (Jain, 1993); VCM de 37.8+3.2 fl (Jain, 1993); hemoglobina de 8.5 a $13.0 \mathrm{~g} / \mathrm{dl}$ (Dürr, 1980) y $11.2 \pm 1.5 \mathrm{~g} / \mathrm{dl}$ (Jain, 1993), cifras aproximadamente coincidentes con las aqui halladas. El hemograma sigue constituyendo el pilar fundamental de las exploraciones de laboratorio, a punto tal que actualmente es utilizado para verificar la normalidad de terneros nacidos de hembras clonadas (Kasai, 2006).

Los leucocitos totales de los animales bajo ensayo resultaron más elevados que los reportados para terneros de razas no índicas, de $10.7 \pm 3.0 \mathrm{G} / 1$ (Jain, 1993) y 4-12 G/l (Dürr, 1980). Los glóbulos blancos registraron una disminución ontogénica

Tabla 1. Valores del peso y hemograma obtenidos para el total de la muestra $(n=60)$.

\begin{tabular}{|c|c|c|c|c|c|}
\hline Variable & Periodo & $\bar{x} \pm D E$ & $\mathrm{IC} \pm 95 \%$ & WS & $T$ \\
\hline \multirow[t]{2}{*}{ peso $(\mathrm{kg})$} & $\operatorname{dia} 0$ & $78,9 \pm 6,9$ & $77,2-80,7$ & 0,987 & $A^{* *}$ \\
\hline & dia 120 & $158,7 \pm 11,7$ & $155,7-161,8$ & 0,986 & \\
\hline \multirow[t]{2}{*}{ eritrocitos $(\mathrm{T} / \mathrm{l})$} & día 0 & $8,97 \pm 0,93$ & $8,72-9,21$ & 0,996 & $\mathrm{D}$ \\
\hline & dia 120 & $8,33 \pm 0,97$ & $8,07-8,58$ & 0,995 & \\
\hline \multirow[t]{2}{*}{ hematocrito $(\%)$} & dia 0 & $38,3 \pm 2,8$ & $37,5-39,1$ & 0,993 & $\mathrm{~A}$ \\
\hline & día 120 & $40,3 \pm 3,0$ & $39.5-41.2$ & 0,995 & \\
\hline \multirow[t]{2}{*}{$\operatorname{VCM}(f)$} & día 0 & $42,7 \pm 4,3$ & $41,5-43,8$ & 0,991 & $A^{*}$ \\
\hline & día 120 & $48,4 \pm 4,3$ & $47,2-49,5$ & 0,997 & \\
\hline \multirow[t]{2}{*}{ hemoglobina (g/dl) } & dia 0 & $12,1 \pm 1,3$ & $11,7-12,4$ & 0,996 & $A$ \\
\hline & dia 120 & $13,8 \pm 1,1$ & $13,3-14,1$ & 0,991 & \\
\hline \multirow[t]{2}{*}{$\mathrm{HCM}(\mathrm{pg})$} & día 0 & $13,9 \pm 1,2$ & $13,5-14,1$ & 0,985 & $A^{*}$ \\
\hline & día 120 & $16,1 \pm 0,9$ & $15,7-16,7$ & 0,975 & \\
\hline \multirow[t]{2}{*}{$\mathrm{CHCM}(\%)$} & día 0 & $31,6 \pm 1,8$ & $31,1-32,0$ & 0,994 & $A^{*}$ \\
\hline & día 120 & $34,2 \pm 1,7$ & $33,7-34,9$ & 0,986 & \\
\hline \multirow[t]{2}{*}{ leucocitos (G/l) } & día 0 & $14,31 \pm 1,69$ & $13,8-14,7$ & 0,986 & $\mathrm{D}^{* *}$ \\
\hline & día 120 & $9,76 \pm 0,90$ & $9,5-9,9$ & 0,977 & \\
\hline \multirow[t]{2}{*}{ neutrófilos $(\mathrm{G} / \mathrm{l})$} & día 0 & $3,58 \pm 0,57$ & $3,42-3,72$ & 0,984 & $A^{*}$ \\
\hline & día 120 & $3,78 \pm 0,59$ & $3,62-3,93$ & 0,991 & \\
\hline \multirow[t]{2}{*}{$\operatorname{linfocitos}(\mathrm{G} / \mathrm{l})$} & día 0 & $10,22 \pm 1,45$ & $9,84-10,60$ & 0,976 & $D^{* *}$ \\
\hline & día 120 & $5,39 \pm 0,76$ & $5,19-5,58$ & 0,989 & \\
\hline \multirow[t]{2}{*}{ monocitos $(\mathrm{G} / \mathrm{l})$} & día 0 & $0,44 \pm 0,08$ & $0,41-0,45$ & 0,981 & D \\
\hline & día 120 & $0,31 \pm 0,09$ & $0,28-0,33$ & 0,993 & \\
\hline \multirow[t]{2}{*}{ eosinófilos (G/l) } & dia 0 & $0,07 \pm 0,05$ & $0,05-0,08$ & 0,948 & $A^{*}$ \\
\hline & dia 120 & $0,36 \pm 0,19$ & $0,32-0,39$ & 0,986 & \\
\hline
\end{tabular}

$\bar{x}$ : media aritmética, DS: desvio estándar, IC: intervalo de confianza, WS: test de normalidad distributiva de WilkShapiro (valor crítico: 0,947$)$, T: tendencia, A: aumento, D: disminución, * significativo $(\mathrm{p}=0,05-0,01)$, ** altamente significativo ( $p<0,01$ ), VCM: volumen corpuscular medio, HCM: hemoglobina corpuscular media, CHCM: concentración de hemoglobina corpuscular media. 
escasamente influenciada por el sexo, similar a la descripta en vacunos de otras latitudes, de 11 a 8 G/1 (Jain, 1993) y de 8 a 7 G/1 (Dürr, 1980). En cambio, no se constató la declinación de neutrófilos ni el aumento de linfocitos reportados para razas lecheras (Jain, 1993). Los monocitos no registraron variaciones significativas y los eosinófilos se elevaron significativamente al aumentar la edad, romo ocurre en otras razas (Jain, 1993). Los basófilos fueron casi inexistentes en los terneros cruza cebú aquí investigados. Además de alterarse en inflamaciones e infecciones, no es infrecuente que los leucocitos de los terneros se afecten por trastornos mieloproliferativos (Nozaki, 2006).

La Tabla 2 muestra los cambios registrados para los electrolitos séricos, así como para una hormona relacionada con la regulación de varios iones circulantes, la aldosterona (Kolb, 1987).

En terneros recién nacidos, de razas europeas y otras latitudes, se hallaron niveles de $533 \pm 159 \mathrm{pg} /$ $\mathrm{ml}$ de aldosterona, que hacia el octavo día de vida habian declinado a $246 \pm 56 \mathrm{pg} / \mathrm{ml}$ (Kaneko, 1989). Ello sugiere una disminución ontogénica de la hormona, tendencia que coincide con la del presente trabajo, aunque los valores aqui obtenidos fueron más altos, quizás debido al clima subtropical y/o la raza índica. Aldosterona es la principal reguladora de sodio, potasio, cloro y agua del medio interno (Coppo, 2001). En el presente ensayo no se registraron cambios significativos de natremia, kalemia ni cloremia ante el avance de la edad de los terneros, cuyos niveles fueron similares a los reportados en ganado adulto (Dürr, 1980; Kolb, 1987; Kaneko, 1989). Ninguno de los tres electrolitos registraria cambios atribuibles al desarrollo ontogénico.

Los terneros bajo ensayo tampoco mostraron concentraciones de calcio, magnesio, hierro y cobre diferentes de los valores consignados para ganado adulto (Dürr, 1980; Kolb, 1987; Kaneko, 1989). En cambio, el fósforo inorgánico reveló valores altos y tendencia decreciente, tal como sucede en razas europeas, donde la fosfatemia del ternero declina desde niveles de 9.5 a $5.5 \mathrm{mg} / \mathrm{dl}$ en vacas (Dürr, 1980). En terneros de 3 meses, otros autores tampoco encontraron diferencias significativas entre sexos para este catión (Ricciardino et al., 1997). El ionograma asume un preponderante rol durante el desarrollo del ternero pues las carencias minerales, especialmente la de cobre, constituyen importantes factores de riesgo para la salud y performance del ternero (Enjalbert, 2006).

En la Tabla 3 se consigna la evolución del proteinograma sérico y de las principales actividades enzimáticas de interés diagnóstico en terneros.

El crecimiento de los terneros se caracterizó por considerables aumentos de proteínas totales y marcada disminución de RAG debido a que, sobre el escaso aumento de albúminas (no significativo) prevalecieron los incrementos de beta globulinas (significativos) y gamma globulinas (altamente significativos). Tales cambios concuerdan con los reportados para otras razas y países por distintos autores (Dürr, 1980; Kolb, 1987; Kaneko, 1989). aseverándose que los niveles de gamma globulinas (anticuerpos) recién alcanzan concentraciones estables a los 6 meses de edad (Jain, 1993). En terneros de otras razas fueron reportadas concentraciones de 3.9 a $6.7 \mathrm{~g} / \mathrm{dl}$ y $6.2 \pm 0.6 \mathrm{~g} / \mathrm{dl}$ para proteínas

Tabla 2. Valores relacionados al metabolismo mineral obtenidos para el total de la muestra $(n=60)$.

\begin{tabular}{|c|c|c|c|c|c|}
\hline Variable & Periodo & $\overline{\bar{x}} \pm \overline{D E}$ & IC $\pm 95 \%$ & WS & $\mathrm{T}$ \\
\hline \multirow[t]{2}{*}{ aldosterona $(\mathrm{pg} / \mathrm{ml})$} & día 0 & $348 \pm 12$ & $345-351$ & 0,996 & $\mathrm{D}^{*}$ \\
\hline & día 120 & $288 \pm 11$ & $285-291$ & 0,995 & \\
\hline \multirow[t]{2}{*}{ sodio $(\mathrm{meq} / \mathrm{l})$} & día 0 & $144 \pm 5$ & $142-145$ & 0,981 & $\mathrm{D}$ \\
\hline & día 120 & $142 \pm 5$ & $140-143$ & 0,980 & \\
\hline \multirow[t]{2}{*}{ potasio $(\mathrm{meq} / \mathrm{l})$} & dia 0 & $4,53 \pm 0,46$ & $4,41-4,64$ & 0,962 & $\mathrm{D}$ \\
\hline & dia 120 & $4,50 \pm 0,49$ & $4,37-4,62$ & 0,973 & \\
\hline \multirow[t]{2}{*}{ cloro (meq/l) } & día 0 & $95,8 \pm 6,8$ & $93,9-97,5$ & 0,975 & A \\
\hline & día 120 & $96,0 \pm 7,4$ & $94,0-97,8$ & 0,969 & \\
\hline \multirow[t]{2}{*}{ hierro (ug/dl) } & día 0 & $109 \pm 17$ & $104-113$ & 0,995 & $\mathrm{~A}$ \\
\hline & día 120 & $112 \pm 17$ & $107-116$ & 0,994 & \\
\hline \multirow[t]{2}{*}{ cobre $(\mathrm{ug} / \mathrm{dl})$} & día 0 & $82 \pm 18$ & $77-86$ & 0,991 & $\mathrm{D}$ \\
\hline & día 120 & $80 \pm 19$ & $75-84$ & 0,992 & \\
\hline \multirow[t]{2}{*}{ fósforo in. (mg/dl) } & día 0 & $9,63 \pm 1,12$ & $9,33-9,92$ & 0,992 & $D^{* *}$ \\
\hline & día 120 & $7,90 \pm 1,04$ & $7,64-8,25$ & 0,996 & \\
\hline \multirow[t]{2}{*}{ magnesio (mg/dl) } & día 0 & $2,47 \pm 0,32$ & $2,38-2,55$ & 0,988 & A \\
\hline & día 120 & $2,56 \pm 0,37$ & $2,46-2,65$ & 0.991 & \\
\hline \multirow[t]{2}{*}{ calcio $(\mathrm{mg} / \mathrm{dl})$} & dia 0 & $9,08 \pm 0,79$ & $8,87-9,29$ & 0,961 & A \\
\hline & día 120 & $9,20 \pm 0,91$ & $8,96-9,43$ & 0,959 & \\
\hline
\end{tabular}

$\overline{\mathbf{x}}$ : media aritmética, DS: desvío estándar, IC: intervalo de confianza, WS: test de normalidad distributiva de WilkShapiro (valor crítico: 0,947$)$, T: tendencia, A: aumento, D: disminución, * significativo $(\mathrm{p}=0,05-0,01),{ }^{* *}$ altamente significativo $(p<0,01)$. 
totales, de $2.9 \mathrm{~g} / \mathrm{dl}$ y 3.0 a $3.6 \mathrm{~g} / \mathrm{dl}$ para albúminas, de $0.90 \mathrm{~g} / \mathrm{dl}$ para alfa globulinas y de $0,80 \mathrm{~g} / \mathrm{dl}$ para beta globulinas, así como de 1 a $2 \mathrm{~g} / \mathrm{dl}$ para gamma globulinas (Corbellini, 1979, Dürr, 1980; Kolb, 1987; Kaneko, 1989; Jain, 1993), muchas de ellas coincidentes con los valores aqui obtenidos.

El aumento de urea sérica paralelo al crecimiento de los terneros bajo ensayo concuerda con datos publicados para otras razas, donde los valores séricos fueron semejantes a los aquí obtenidos, de 0.10-0.40 g/l, sin diferencias significativas entre sexos (Dürr, 1980).

En el presente trabajo, los valores de ALP en animales de cruza índica resultaron semejantes a los hallados en terneros de otras razas y regiones, de 309 UI/l (Fagliari et al., 1996) y 315 UI/l (Dürr, 1980). En todos los mamíferos, esta enzima asume actividades altas en la joven edad, debido al crecimiento óseo (Coppo, 2001). Los animales bajo ensayo no escaparon de esta premisa, registrando una declinación ontogénica altamente significati$\mathrm{va}$, coincidente con resultados obtenidos en otras investigaciones, donde ALP disminuyó desde niveles superiores a $300 \mathrm{UI} / \mathrm{l}$ en terneros, hasta 200 UI/l en adultos (Dürr, 1980).

Para las actividades de AST, LDH y GGT, explo- radoras de la función hepática (Coppo, 2001), no se registraron cambios atribuibles al crecimiento y sus valores séricos resultaron semejantes a los reportados para terneros en otras investigaciones (Kolb, 1987; Kaneko, 1989), aunque algunos autores hallaron actividades de LDH mucho más elevadas en razas europeas, de hasta $1450 \mathrm{UI} / \mathrm{l}$ (Dürr, 1980).

En cambio, CPK se elevó significativamente en función del crecimiento del ternero, probablemente por el aumento de su masa muscular, principal tejido de origen de esta enzima (Kaneko, 1989). Los valores aqui obtenidos para cruzas índicas fueron sensiblemente más altos que los reportados para razas británicas, de 30-50 UI/I (Dürr, 1980). CPK es la enzima de elección para tipificar diversas miopatías del ternero (Pradhan, 2006).

En la Tabla 4 se detallan los resultados obtenidos para los parámetros glucídicos y lipidicos, así como los de una hormona que interviene en su regulación homeostática, el cortisol (Coppo, 2001).

El aumento ontogénico aquí registrado para cortisol concuerda con reportes que aseveran el incremento de esta hormona en otras razas bovinas, desde la etapa de lactación ( $1-3 \mathrm{ug} / \mathrm{dl})$ hasta alcanzar en la adultez niveles de 5-15 ug/dl (Gómez Pi-

Tabla 3. Valores relacionados al metabolismo proteico y enzimas obtenidos para el total de la muestra $(\mathrm{n}=60)$.

\begin{tabular}{|c|c|c|c|c|c|}
\hline Variable & Periodo & $\bar{x} \pm D F$ & $\mathrm{IC} \pm 95 \%$ & WS & $\mathrm{T}$ \\
\hline \multirow[t]{2}{*}{ proteinas tot. $(\mathrm{g} / \mathrm{dl})$} & $\operatorname{dia} 0$ & $5,73 \pm 0,34$ & $5,64-5,81$ & 0,948 & $A^{* *}$ \\
\hline & día 120 & $6,91 \pm 0,38$ & $6.80-7,01$ & 0.950 & \\
\hline \multirow[t]{2}{*}{ albúmina $(\mathrm{g} / \mathrm{d} l)$} & $\operatorname{dia} 0$ & $3,29 \pm 0.28$ & $3,21-3,36$ & 0.949 & $\mathrm{~A}$ \\
\hline & dia 120 & $3,39 \pm 0,29$ & $3.27-3.42$ & 0.962 & \\
\hline \multirow[t]{2}{*}{ alfa globul. (g/dl) } & día 0 & $0,75 \pm 0,13$ & $0,72-0,79$ & 0,982 & A \\
\hline & día 120 & $0.79 \pm 0.13$ & $0,75-0,82$ & 0,991 & \\
\hline \multirow[t]{2}{*}{ beta globul. (g/dl) } & día 0 & $0,79 \pm 0,16$ & $0,74-0,82$ & 0,975 & $A^{*}$ \\
\hline & dia 120 & $0,82 \pm 0,11$ & $0,78-0,85$ & 0,927 & \\
\hline \multirow[t]{2}{*}{ gamma globul.(g/dl) } & dia 0 & $0,88 \pm 0,13$ & $0,85-0,92$ & 0,991 & $A^{* *}$ \\
\hline & día 120 & $1,91 \pm 0,28$ & $1,88-2,04$ & 0,977 & \\
\hline \multirow[t]{2}{*}{ RAG (score) } & dia 0 & $1,38 \pm 0,16$ & $1,33-1,41$ & 0,986 & $D^{*}$ \\
\hline & dia 120 & $0,94 \pm 0,15$ & $0,90-0,98$ & 0,992 & \\
\hline \multirow[t]{2}{*}{ urea $(g / l)$} & día 0 & $0,22 \pm 0,03$ & $0,21-0,23$ & 0,968 & $A^{*}$ \\
\hline & día 120 & $0,30 \pm 0,04$ & $0,29-0,33$ & 0,949 & \\
\hline \multirow[t]{2}{*}{ ALP (UI/1) } & día 0 & $453 \pm 51$ & $439-466$ & 0,995 & $D^{* *}$ \\
\hline & dia 120 & $331 \pm 30$ & $323-339$ & 0,994 & \\
\hline \multirow[t]{2}{*}{$\operatorname{AST}(\mathrm{UI} / \mathrm{l})$} & día 0 & $31,8 \pm 7,8$ & $29,7-33,8$ & 0,989 & $\mathrm{D}$ \\
\hline & día 120 & $30,6 \pm 8,1$ & $28,4-32,6$ & 0,981 & \\
\hline \multirow[t]{2}{*}{$\mathrm{LDH}(\mathrm{UL} / \mathrm{l})$} & dia 0 & $597 \pm 127$ & $564-630$ & 0,958 & $\mathrm{D}$ \\
\hline & día 120 & $584 \pm 126$ & $550-616$ & 0,965 & \\
\hline \multirow[t]{2}{*}{ GGT (UI/l) } & día 0 & $14,8 \pm 6,9$ & $13,0-16,5$ & 0,964 & $\mathrm{D}$ \\
\hline & día 120 & $14,6 \pm 6,6$ & $12,8-16,3$ & 0,953 & \\
\hline \multirow[t]{2}{*}{ CPK (UI/l) } & dia 0 & $111 \pm 28$ & $103-118$ & 0,956 & $A^{*}$ \\
\hline & día 120 & $170 \pm 33$ & $161-178$ & 0,987 & \\
\hline
\end{tabular}

$\overline{\mathrm{x}}$ : media aritmética, DS: desvio estándar, IC: intervalo de confianza, WS: test de normalidad distributiva de WilkShapiro (valor crítico: 0,947), T: tendencia, A: aumento, D: disminución, ${ }^{*}$ significativo $(\mathrm{p}=0,05-0,01),{ }^{* *}$ altamente significativo ( $p<0,01$ ), globul.: globulinas, RAG: relación albúmina/globulinas, ALP: fosfatasa alcalina, AST: aspartato aminotransferasa, LDH: lactato dehidrogenasa, GGT: gamma glutamiltransferasa, CPK: creatinfosfoquinasa. 
quer, 1992), 3-7 ug/dl (Kaneko, 1989) o 10 \pm 4 ug/dl (Domíngues et al., 1997).

La glucemia siempre es más alta en terneros $(0,80$ $1.20 \mathrm{~g} / \mathrm{l}$, Dürr, 1980); su descenso hacia valores normales de adulto comenzaria a partir de la octava semana de vida (Gómez Piquer, 1992). Cuando el metabolismo del animal cambia de monogástrico a rumiante, la glucemia disminuye un $50 \%$, hasta 0,4-0,7 g/l (Kolb, 1987). Tal declinación coincide con la observada en el presente ensayo, no asi los elevados valores iniciales aqui obtenidos (cercanos a $2 \mathrm{~g} / \mathrm{l}$ en algunos casos), quizás debido a que la indocilidad de la cruza indica provoca mayor alarma simpática durante la manipulación y consecuente hiperglucemia por elevación episódica de catecolaminas (Coppo, 2001).

La fructosamina es el compuesto resultante de la fijación de glucosa a grupos amino libres de proteinas plasmáticas. Dado que la magnitud de la glicosilación depende de los niveles promedios de glucemia, esta glicoproteína constituye una excelente indicadora de hiperglucemia crónica, permitiendo descartar aquéllas causadas por alarmas fugaces (Jensen, 1993), como las ocurridas en este trabajo. No se hallaron referencias bibliográficas sobre fructosaminemia en terneros; su nivel en bovinos adultos sería de 213-265 umol/l (Jensen, 1993). Los más altos valores aqui registrados deberian interpretarse en función de las más elevadas glucemias de los terneros (Coppo, 2002). Por ello, el descenso de fructosamina debe ser considerado como un cambio ontogénico del ternero.

Triglicéridos y colesterol descendieron significati- vamente ante el avance de la edad de los animales, seguramente debido a la reducción del aporte de grasas lácteas en función del aumento del consumo de pasturas. La lipemia sería influida considerablemente por el contenido graso de la dieta, aumentando cuando el alimento es rico en lípidos, como la leche (Kolb, 1987). Los valores de triglicéridos son más bajos en el bovino adulto en pastoreo, de 0,15 a $0,30 \mathrm{~g} / 1$ (Dürr, 1980). Los valores aqui obtenidos para colesterol total (superiores a 1 g/l) difieren considerablemente de los reportados para terneros europeos de razas carniceras, de 0,3$0,4 \mathrm{~g} / \mathrm{l}$ (Dürr, 1980).

En nuestra experiencia, los niveles de C-HDL mostraron una declinación ontogénica paralela a la de su lipoproteina transportadora (alfa), en tanto que las concentraciones de C-LDL aumentaron concomitantemente con la fracción lipoproteica a la cual está ligado (beta). No se discuten los valores de C-HDL, C-LDL y lipoproteínas alfa y beta porque los escasos datos hallados en la bibliografía no son comparables por haber sido obtenidos con otras técnicas de laboratorio.

\section{CONCLUSIONES}

Se establecen intervalos de confianza y se revelan variaciones ontogénicas de varios parámetros hemáticos de interés diagnóstico, registrados en terneros sanos cruza cebú mantenidos en lactación al pie de madre sobre pasturas naturales del nordeste argentino. Entre los 2 y 6 meses de edad, se verifican $(p<0,05)$ aumentos significativos de cortisol, CPK, VCM, HCM, CHCM, neutrófilos, eosinófi-

Tabla 4. Valores relacionados a los metabolismos glucídico y lipídico obtenidos para el total de la muestra $(n=60)$.

\begin{tabular}{|c|c|c|c|c|c|}
\hline Variable & Período & $\bar{x} \pm D E$ & {$[C \pm 95 \%$} & WS & $\mathrm{T}$ \\
\hline \multirow{2}{*}{ cortisol (ug/dl) } & dia 0 & $2,2 \pm 0,5$ & $2,1-2,3$ & 0,951 & $A^{*}$ \\
\hline & día 120 & $3,4 \pm 0,8$ & $3,1-3,7$ & 0,995 & \\
\hline \multirow[t]{2}{*}{ glucosa $(\mathrm{g} / \mathrm{l})$} & día 0 & $1,52 \pm 0,33$ & $1,43-1,60$ & 0,979 & $D^{* *}$ \\
\hline & día 120 & $0,94 \pm 0,15$ & $0,90-0,97$ & 0,978 & \\
\hline \multirow[t]{2}{*}{ fructosam. (umol/l) } & día 0 & $297 \pm 35$ & $294-302$ & 0,974 & $D^{* *}$ \\
\hline & dia 120 & $226 \pm 33$ & $217-234$ & 0,991 & \\
\hline \multirow[t]{2}{*}{ triglicéridos $(\mathrm{g} / \mathrm{l})$} & día 0 & $0,42 \pm 0,13$ & $0,38-0,45$ & 0,994 & $\mathrm{D}^{*}$ \\
\hline & día 120 & $0,36 \pm 0,1 C$ & $0,33-0,38$ & 0,985 & \\
\hline \multirow[t]{2}{*}{ colesterol total $(\mathrm{g} / \mathrm{l})$} & dia 0 & $1,15 \pm 0,32$ & $1,07-1,23$ & 0,959 & $D^{*}$ \\
\hline & día 120 & $1,07 \pm 0,29$ & $0,99-1,14$ & 0,988 & \\
\hline \multirow[t]{2}{*}{ C-HDL (g/l) } & día 0 & $0,78 \pm 0,13$ & $0,75-0,81$ & 0,994 & $\mathrm{D}$ \\
\hline & dia 120 & $0,70 \pm 0,12$ & $0,66-0,72$ & 0,995 & \\
\hline \multirow[t]{2}{*}{ C-LDL $(\mathrm{g} / \mathrm{l})$} & día 0 & $0,21 \pm 0,09$ & $0,18-0,22$ & 0,975 & $\mathrm{~A}$ \\
\hline & día 120 & $0,24 \pm 0,08$ & $0,21-0,26$ & 0,978 & \\
\hline \multirow[t]{2}{*}{ lipoprot. alfa (\%) } & dia 0 & $83,7 \pm 6,6$ & $81,9-85,4$ & 0,951 & $\mathrm{D}$ \\
\hline & día 120 & $82,9 \pm 6,3$ & $81,2-84,5$ & 0,976 & \\
\hline \multirow[t]{2}{*}{ lipoprot. beta (\%) } & día 0 & $16,3 \pm 6,4$ & $14,6-17,9$ & 0,953 & A \\
\hline & día 120 & $17,1 \pm 5,9$ & $15,5-18,6$ & 0,987 & \\
\hline
\end{tabular}

$\overline{\mathrm{x}}$ : media aritmética, DS: desvío estándar, IC: intervalo de confianza, WS: test de normalidad distributiva de WilkShapiro (valor crítico: 0,947), T: tendencia, A: aumento, $\mathrm{D}$ : disminución, ${ }^{*}$ significativo $(\mathrm{p}=0,05-0,01),{ }^{* *}$ altamente significativo $(\mathrm{p}<\mathbf{0 , 0 1})$, fructosam.: fructosamina, lipoprot.: lipoproteinas. 
los, urea, proteínas totales, beta y gamma globulinas. En cambio, se producen disminuciones significativas de aldosterona, fructosamina, glucosa, triglicéridos, colesterol total, fósforo inorgánico, ALP, RAG, leucocitos totales y linfocitos. Algunos de estos cambios difieren con los observados en razas europeas. Comparados con los reportados para otros paises y razas, los terneros con sangre índica de dos meses de edad, poseen concentraciones más altas de aldosterona, $\mathrm{CPK}$, leucocitos totales, neutrófilos, glucosa y colesterol total, así como niveles más bajos de LDH y linfocitos.

Agradecimientos. El trabajo se realizó con subsidios de SGCYT-UNNE (17B/041) y CONICET (PIP 577). Se agradece la colaboración prestada por los Dres. A. Slanac, M. Revidatti y A. Capellari.

\section{BIBLIOGRAFÍA}

Ash, K.O. 1980. Reference intervals: a challenge to laboratorians. Am. J. Med. Technol. 46: 504-51I.

Coppo, J.A. 1999. Comparación entre hemogramas de terneros destete obtenidos por métodos manuales versus automatizados. Rev. Vet. 8: 43-46.

Coppo. J.A. 2001. Fisiología Comparada del Medio Interno, Ed. Dunken, Buenos Aires, 300 p.

Coppo, J.A. 2002. Evolution of fructosaminaemia and glucaemia during the growth of unweaned and early weaned half-bred Zebu calves. Vet. Res. Comm. 25: 449-459.

Corbellini, C. 1979. Proteinogramas en terneros calostrados y no calostrados. Med. Vet. 60: 216-221.

Domingues, P.F.; Oba, E.; Uribe, L.F.; Souza, M.I.; Ramos, A.A. 1997. Concentraçao plasmática de cortisol em vacas com mastite subclínica. Anales XXV Congr. Brasil. Med. Vet., Gramado (Brasil), Comunic. REP 024.

Dürr, U.M. 1980. Laboratory Testing in Veterinary Medicine. Ed. B. Mannheim, Munich, 130 p.

Enjalbert, F. 2006. Effects of copper, zinc and selenium status on performance and health in commercial dairy and beef herds. J. Anim. Physiol. 90: 459-466.

Fagliari, J.J.; Oliveira, E.C.; Pegorer, M.F.; Ferrante, L.C.; Campos Filho, E. 1996. Relaçao entre nivel sérico de gamaglobulinas e as atividades de GGT, ALP e AST de bezerros recém-nascidos. Anales
XXIV Congr. Brasil. Med. Vet., Goiania, Brasil, p. 75.

Foote, M.R.; Nonnecke, B.J.; Beitz, D.C.; Waters, W.R. 2007. High growth rate fails to enhance adaptive immune responses of neonatal calves. J. Dairy Sci. 90: 404-417.

Gómez Piquer, J. 1992. Manual Práctico de Análisis Clínicos Veterinarios, Ed. Mira, Zaragoza, 445 p.

Jain, N.C. 1993. Essentials of Veterinary Hematology, Ed. Lea \& Febiger, Philadelphia, 417 p.

Jensen, A.L. 1993. Determination of fructosamine concentration in bovine serum samples. J. Vet. Med. A. 40: 111-117.

Kaneko, J.J. 1989. Clinical Biochemistry of Domestic Animals, Ed.Academic Press, San Diego, 832 p.

Kasai, K. 2006. Comparison of the growth performances of offspring produced by a pair of cloned cattle and their nuclear donor animals. J. Reprod. Dev. 52: 711-715.

Kolb, E. 1987. Fisiología Veterinaria, $3^{\circ}$ ed., Ed. Acribia, Zaragoza, $1115 \mathrm{p}$.

Nozaki, S. 2006. Natural killer-like T-cell lymphoma in a calf. J. Comp. Pathol. 135: 47-51.

Peruchena, C.O. 1992. Nutrición de bovinos sobre pastizales de baja calidad de la región NEA. Anales de las I Jornadas de Nutrición Bovina, Facultad de Ciencias Veterinarias UNNE, Corrientes, p. 22.

Peruchena, C.O. 1996. Destete precoz, manejo y nutrición de los terneros. Anales de la Jornada Técnica sobre Destete Precoz, INTA EEA Corrientes, p. 1-18.

Pradhan, S. 2006. Calf-head sign in miyoshi myopathy. Arch. Neurol. 63: 1414-1417.

Ricciardino, M.Z.; Scena, C.; Piccinali, R.L. 1997. Relaciones entre parámetros bioquimicos y productivos en terneros lactantes de vacas primíparas Hereford. Rev. Arg. Prod. Anim. 17: 1, 305.

Solberg, H.E. 1992. Teoría de los valores de referencia. Acta Bioq. Clin. Lat. 26: 105-110.

Steel, R.G.; Torrie, J.H. 1992. Principles and Procedures of Statistics. A Biometrical Approach. 2nd. ed., Ed. McGraw-Hill, New York, 715 p.

Zehentner, O. 1996. Experiencias de destete precoz en la Provincia de Misiones. Anales de la Jornada Técnica sobre Destete Precoz, INTA EEA Corrientes, p. 31-42. 